\title{
OPEN Regulation of PDE5 expression in human aorta and thoracic aortic aneurysms
}

Received: 1 April 2019

Accepted: 25 July 2019

Published online: 21 August 2019

\author{
Valeriana Cesarini ${ }^{1,2}$, Calogera Pisano $\mathbb{D}^{3}$, Gabriele Rossi ${ }^{1}$, Carmela Rita Balistreri ${ }^{4}{ }^{4}$, \\ Flavia Botti ${ }^{5}$, Giorgio Antonelli ${ }^{1}$, Giovanni Ruvolo ${ }^{2}$, Emmanuele A. Jannini ${ }^{6}{ }^{6}$ \& Susanna Dolci ${ }^{1}$
}

Aneurysms and dissections affecting thoracic aorta are associated with smooth muscle cell (SMC) dysfunction. NO/cGMP signaling pathway in smooth muscle cells has been shown to be affected in sporadic thoracic aortic aneurysms. We analyzed the mRNA levels of PDE5, a cGMP-hydrolyzing enzyme highly expressed in aortic SMCs, that regulates arterious vascular tone by lowering cGMP levels. We found that aortic tissue obtained from Marfan, tricuspid and bicuspid thoracic aneurysms expressed lower levels of PDE5 mRNA compared to control aortas. In particular, we found that affected aortas showed lower levels of all the PDE5A isoforms, compared to control aortas. Transfection of vascular SMCs (VSMCs) with NOTCH3 activated domain (NICD3) induced the expression of PDE5A1 and A3 protein isoforms, but not that of the corresponding mRNAs. VSMC stimulation with GSNO, a nitric oxide analogue or with 8-br-cGMP, but not with 8-br-cAMP, up-regulated PDE5 and NOTCH-3 protein levels, indicating a negative feedback loop to protect the arterial wall from excessive relaxation. Finally, we found that PDE5 is expressed early during human aorta development, suggesting that if loss of function mutations of PDE5 occur, they might potentially affect aortic wall development.

Thoracic aortic aneurysm (TAA) and dissection (TAD) remain a significant clinical challenge due to their high morbidity and mortality ${ }^{1,2}$. In the United States, diseases of the aorta account for more than 40.000 deaths per year $^{3}$. The aetiology of TAA is heterogeneous and can be classified in syndromic and non-syndromic forms. The first includes inherited and familiar TAA that characterizes younger patients. The second group includes sporadic or isolated TAAs, that occur with aging. Inherited TAAs have an incidence of $5 \%$ and are represented by Marfan syndrome, Ehlers-Danlos syndrome, Loeys-Dietz and aneurysm-osteoarthrosis syndrome ${ }^{4}$. Within the TAAs, the familiar forms have a $20 \%$ incidence and are represented by TAA associated with bicuspid aortic valve (BAV), patent ductus arteriosus (PDA) and cerebro-vascular disease. Isolated or sporadic TAAs, accounting for the remaining $75 \%$, have a degenerative etiology linked to classic cardiovascular risk factors, such as smoking, hypertension, and dyslipidemia ${ }^{5,6}$ and are defined as tricuspid aortic valve aneurysms (TAV). Recent studies showed a genetic basis of all forms of TAA even in the sporadic type ${ }^{7}$. It is predominantly inherited in an autosomal dominant fashion with reduced penetrance and variable expression, showing significant genetic ( $>20$ gene) and clinical (location, severity and age onset) heterogeneity ${ }^{8}$. Most genes associated with the development of TAA encode proteins involved in the extracellular matrix maintenance, SMC contraction or metabolism, or in signaling pathways ${ }^{9}$. The causative genes have been grouped into several categories: structural and regulatory proteins involved in SMC contractile function ${ }^{10-14}$, proteins involved in the maintenance of or SMC adhesion to the extracellular matrix ${ }^{12,15}$, proteins involved in canonical TGF- $\beta$ or NOTCH1 signaling ${ }^{16-23}$ and proteins involved in SMC metabolism or survival ${ }^{14,24}$. Sporadic TAAs have been linked to altered cGMP activated pathway and deregulated nitric oxide synthase (NOS) has been involved in TAA degeneration ${ }^{25-27}$. In addition, a single heterozygous mutation in the gene encoding cGMP-dependent protein kinase 1 (PRKG1; protein PKG-1; chromosomal 10q11.2-q21.1) has been demonstrated to cause sporadic TAA. PKG-1 is activated on binding of cGMP and plays an important role

\footnotetext{
${ }^{1}$ Department of Biomedicine and Prevention, University of Rome TorVergata, Rome, Italy. ${ }^{2}$ Saint Camillus International University of Health and Medical Sciences, Rome, Italy. ${ }^{3}$ Department of Surgery Sciences, University of Rome TorVergata, Rome, Italy. ${ }^{4}$ Department of Biomedicine, Neuroscience and Advanced Diagnostics (Bi.N.D.), University of Palermo, Palermo, Italy. ${ }^{5}$ Department of Clinical Sciences and Translational Medicine, University of Rome TorVergata, Rome, Italy. ${ }^{6}$ Department of Systems Medicine, University of Rome TorVergata, Rome, Italy. Emmanuele A. Jannini and Susanna Dolci jointly supervised this work. Correspondence and requests for materials should be addressed to S.D. (email: dolci@uniroma2.it)
} 
in SMC relaxation. The causal mutation, pArg177Gln, abolishes binding of cGMP and constitutively activates PKG1. Individuals harbouring this mutation have TAD at a relatively young age (15-51 years) at diameter ranging 4.3 to $5.7 \mathrm{~cm}^{14,28}$

Under physiological conditions, cGMP signaling is strictly modulated by cGMP synthesis and inactivation. This latter is carried out by cGMP-phoshodiesterases (cGMP-PDEs) that can specifically hydrolize cGMP or both cGMP and cAMP ${ }^{29}$. The most abundant cGMP-PDEs present in arterial smooth muscle are PDE1A, 1B, and $1 \mathrm{C}, \mathrm{PDE} 3 \mathrm{~A}$ and $3 \mathrm{~B}$, and PDE5 ${ }^{29}$. Of these enzymes, PDE5 has attracted the attention of researchers during the recent years, because it represents the recognized target in treating erectile dysfunction (ED) and several other dysfunctions and symptoms. PDE5 is highly expressed in smooth muscle cells of vessels and as for other PDEs, three isoforms have been identified in humans including PDE5A1, PDE5A2, and PDE5A $3^{30-32}$. These RNAs differ in the $5^{\prime}$ terminus of the respective mRNAs and the transcripts originate from three alternative first exons in the pre-mRNA. All the three isoforms, that show differential amino acid lengths (PDE5A1 is 875, PDE5A2 is 833, and PDE5A3 is 823 amino acid long) have the same cGMP catalytic activity and are sensitive to PDE5 specific inhibitors ${ }^{33}$

NOTCH superfamily contains four members, NOTCH1, NOTCH2, NOTCH3 and NOTCH4, that encode for transmembrane proteins that activate an intracellular signaling pathway through interactions with adjacent ligand expressing cells that lead to the regulation of cell survival, proliferation and differentiation ${ }^{34}$. $\mathrm{NOTCH}$ signaling plays an essential role in the regulation of smooth muscle differentiation, and mutations or haploinsufficiency of NOTCH1 or NOTCH3 have been shown to be involved in a subset of TAA ${ }^{35,36}$ or in cerebral aneurysms, respectively. NOTCH1 signaling has been shown to be positively influenced by cGMP levels and PDE5 inhibition by sildenafil increases stemness of glioma cancer initiating cells in vitro and in vivo ${ }^{37}$. Considering the role of PDE5 in regulating the level of cGMP in smooth muscle cells, in this pilot study we sought to analyse PDE5, NOTCH1 and NOTCH3 expression in TAA patients with Marfan's Syndrome, BAV and TAV. We also studied if NOTCH overexpression can affect PDE5 expression in vitro in smooth muscle cells and in glioma cells. Finally, to understand if PDE5 can play a role in aorta development, we studied its ontogenetic expression profile during early fetal development, both in mice and humans.

\section{Results}

PDE5 is the most abundant CGMP-PDE in adult human aortas and its expression is strongly down-regulated in TAAs. Since cGMP metabolism defects are associated with familial TAA syndromes and PDE5 is highly expressed in SMCs of the aortic wall, we hypothesized that a correlation between PDE5 expression and medial defects might exist. By qRT-PCR we evaluated total PDE5 mRNA levels in TAV, BAV and Marfan syndrome TAA samples and compared them to control aortas. Normalization of PDE5 mRNA was referred either to smooth muscle actin (SMA) mRNA levels, since PDE5 is expressed exclusively in smooth muscle cells and their number could be altered in TAAs or to GAPDH. As shown in Fig. 1 we found that PDE5 mRNA levels were strongly decreased in all the three pathological conditions compared to control aortas (Fig. 1A,B) either when normalizing for SMA or for GAPDH levels. Moreover, any statistically significant difference of the decrease of PDE5 levels among the three types of TAA samples could be observed (Fig. 1A,B). Accordingly, by immunohistochemistry we found that PDE5 staining in the medial layer of the aortic samples was significantly decreased in all the three pathologies compared to control samples (Fig. 1C). As a negative control for PDE5 immunohistochemistry we omitted the primary antibody in the staining procedure (Suppl. Fig. 1E). PDE5A expression is characterized by the presence of three isoforms, A1, A2 and A3, whose expression has not very well characterized in human tissues. While PDE5A1 and $A 2$ first exons are alternative exons, PDE5 A3 first exon spans the first intron and second exon of PDE5 A1 isoform. To correctly analyze their expression in control and aneurysm aortas, we performed qRT-PCR analysis using isoform specific forward primers (exon 1 sequence for the PDE5A1 isoform, alternative exon 1 sequence for the PDE5A2 isoform and intron1/exon2 boundary sequence of for the PDE5A3 isoform) and a common reverse primer within exon 3 . We found that all the three PDE5 isoforms (PDE5A1, A2, A3) were decreased in the pathologic samples compared to control aortas (Fig. 1D).

We also evaluated the expression levels of other cGMP-hydrolizing phosphodiesterases such as PDE1A, $P D E 2 A$ PDE3A and PDE9 in control and TA aortas, however their levels were at the limit of the detection threshold in all the tissue analyzed and thus not assayable (not shown).

NOTCH1 and NOTCH3 levels expression in TAAs. The regulation of PDE5 expression is still an open question and no results have been reported to date. Thus, we investigated if PDE5 expression might be correlated to the expression of transcription factors that are involved in aortic aneurysm and/or smooth muscle development. To this end, we identified two components of the NOTCH family, NOTCH1 and NOTCH3, whose mutations are involved in abnormal VSMCs development ${ }^{38-40}$. By quantitative RT-PCR analysis we found that NOTCH3 levels were increased in Marfan and in TAV samples but not in BAV samples (Fig. 2A). Also NOTCH1 levels were increased in Marfan syndrome samples, however they were similar to control samples in BAV and TAV samples (Fig. 2B). We noticed that one of out $12 \mathrm{BAV}$ samples was completely negative for NOTCH1 expression, but not for $\mathrm{NOTCH}$, and separated it from the remaining BAV samples (not shown). Since the major source of NOTCH1 expression comes from the endothelial compartment of the arterial wall, it is possible that a reduced contribution of endothelium in this sample was the cause of reduced NOTCH1, but not NOTCH3 levels.

PDE5 expression is up-regulated by NOTCH3 and down-regulated by NOTCH1. Since we found that NOTCH1 and NOTCH3 levels are differentially expressed in the aortic aneurysm samples, we next evaluated if they might regulate PDE5 expression in VSMCs in vitro. To this end we performed overexpression experiments by transfecting the intracellular domain of NOTCH1 (NICD1) or NOTCH3 (NICD3) in uterine VSMC. We found that NICD1 transfection induced a significant decrease of PDE5 protein levels in VSMCs (Fig. 3A) 


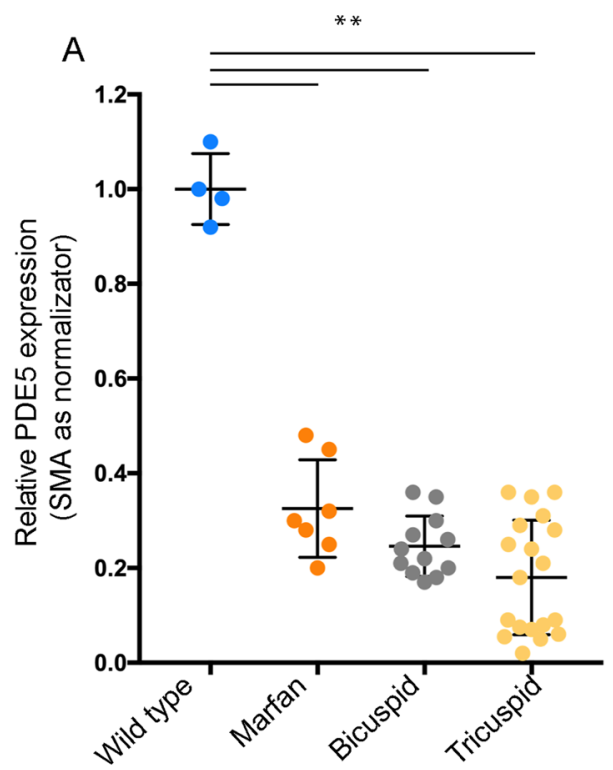

C
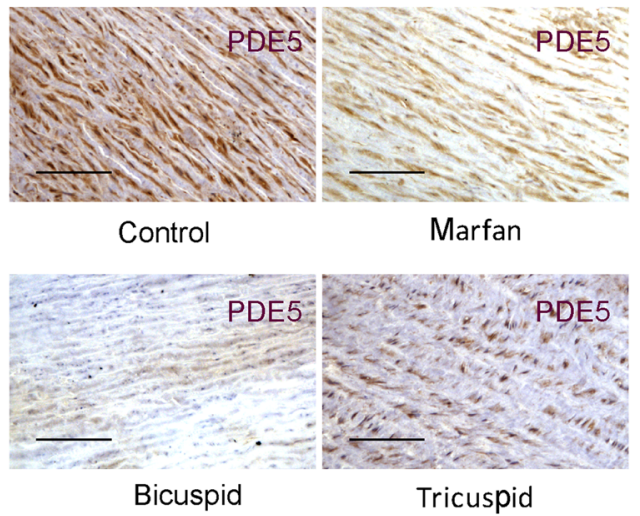

B
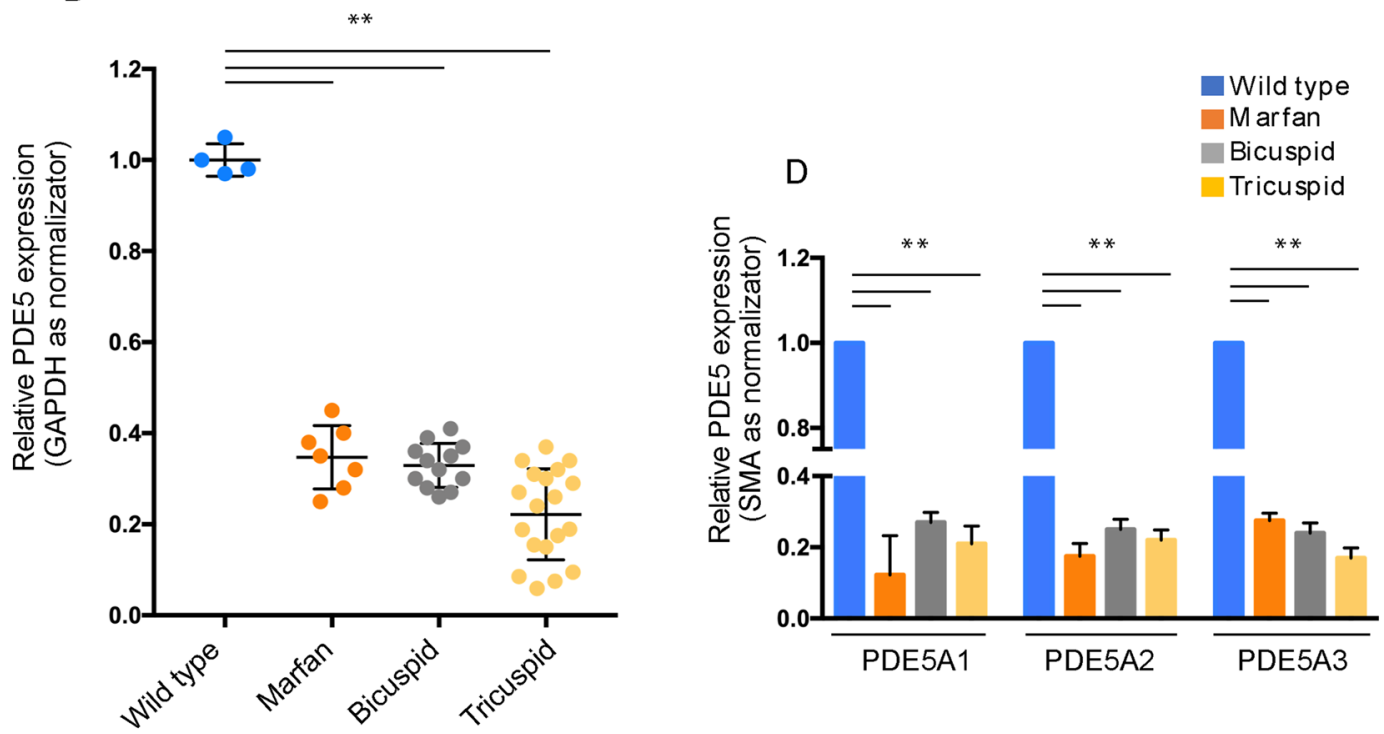

Figure 1. PDE5 expression in control aortas and in TAAs samples. Quantitative RT-PCR showing PDE5 mRNA levels downregulation in TAV, BAV and Marfan syndrome TAA samples compared to control aortas. Normalization of PDE5 mRNA levels was referred to SMA (A) and GAPDH (B) mRNA levels. Data were normalized to the mean of control values, set to 1.0. (C) Representative images of PDE5 expression in the aortic medial layer of control, Marfan, BAV and TAV samples by immunoistochemistry. Bars $=50 \mu \mathrm{m}$ (D) Quantitative RT-PCR of PDE5A1, A2 and A3 isoforms in controls and TAA specimens. Bars represent \pm SD. $* * \mathrm{p}<0.01$ on three experiments.

while NICD3 overexpression, on the contrary, induced a significant up-regulation of the intensity of PDE5 A1 band (Fig. 3A) and the appearance of an extra band of approximately $85 \mathrm{KDa}$ of molecular weight (MW), compatible with the MW of the PDE5A3 isoform. Similarly to VSMCs, also U87MG cells, a glioblastoma cell line that expresses low levels of PDE5, up-regulated both the A1 and A3 isoforms when transfected with NOTCH3 (Fig. 3B), suggesting a common mechanism of NICD3 action on PDE5 expression in smooth muscle and neural cell types. Thus, to understand if $\mathrm{NOTCH} 1$ and/or NOTCH3 were acting at transcriptional level, we evaluated PDE5 mRNA levels in NICD1 or NICD3 overexpressing VSMCs. As shown in Fig. 3C we found that the mRNA levels for PDE5A1, A2, A3 isoforms and for total PDE5 did not significantly change following NICD1 or NICD3 transfection, indicating that their opposing effects on PDE5 protein levels were not occurring at the transcriptional level in VSMCs.

An increase of intracellular cGMP but not cAMP induces PDE5 up-regulation. Both on demand and chronic use of PDE5 inhibitors to treat erectile dysfunction or pulmonary hypertension, that lead to the 
A

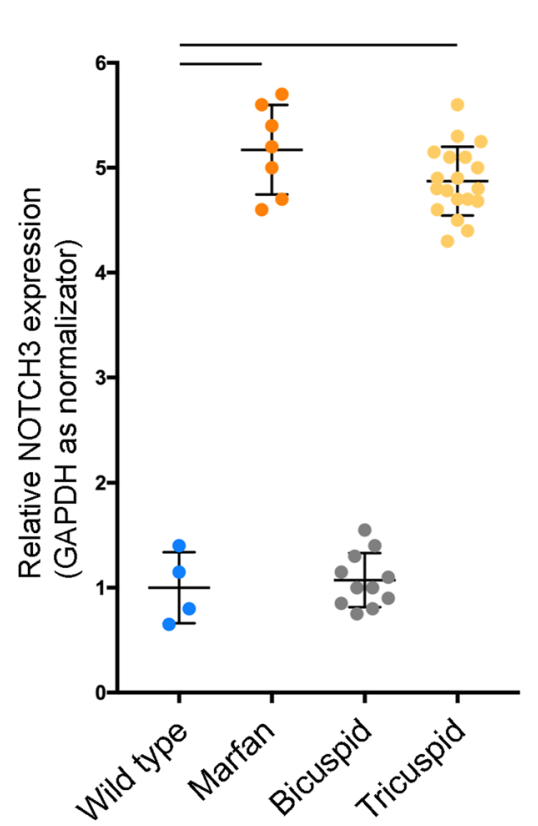

B

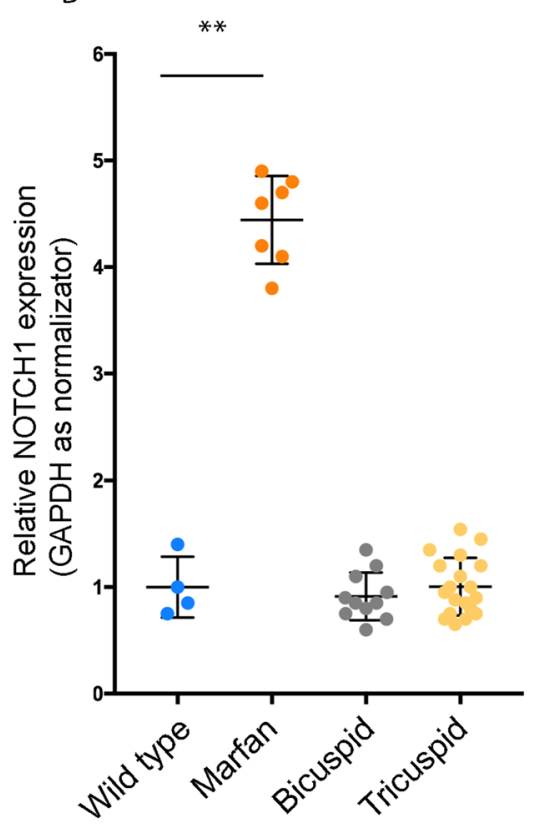

Figure 2. NOTCH1 and NOTCH3 expression levels in TAAs. Quantitative RT-PCR analysis showing NOTCH3 mRNA levels increase in Marfan and in TAV samples compared to BAV samples and control aortas (A) and NOTCH1 mRNA levels increase in Marfan syndrome samples compared to BAV, TAV and control samples (B). Data were normalized to the mean of control values, set to 1.0 . Bars represent \pm SD. ${ }^{*} \mathrm{p}<0.01$ on three experiments.

increase of intracellular cGMP levels, has raised potential safety issues for the rupture of pre-existing aneurysms ${ }^{41}$. Since PDE5 expression is low in aortic aneurysms, we tested if high levels of intracellular cGMP were able to modulate PDE5 expression. We treated VSMCs from human or rat (RCC) origin for $24 \mathrm{hr}$ with $0.5 \mathrm{mM} 8$ - $\mathrm{Br}-\mathrm{cGMP}$ or with $0.5 \mathrm{mM} 8$-Br-cAMP (membrane-permeable analogs of the second messengers cGMP and cAMP, respectively) and in parallel experiments we stimulated VSMCs with $100 \mathrm{nM}$ GSNO, a NO donor that stimulates the soluble guanylate cyclase to produce cGMP. As shown in Fig. 4, we found that 8-br-cGMP or GSNO sensibly up-regulated PDE5 expression while 8-Br-cAMP, that is physiologically generated by $\beta_{2}$-adrenergic receptor stimulation of the medial layer, down-regulated PDE5 levels both in human and rat smooth muscle cells. We also found that NICD1 and NICD3 were up-regulated in human VSMCs. However NICD3, similarly to PDE5, was significantly down-regulated by high intracellular cAMP levels.

Developmental expression of PDE5 in mouse and human fetal aortas. Despite the plethora of data on PDE5 expression and activity in normal and pathological human adult tissues, data are missing on its expression in human or even in animal model embryos. To study the tissue distribution and the developmental stage of PDE5 expression and to co-localize it with NOTCH3 during early development, we performed PDE5 and NOTCH3 IHC experiments on early mouse and human embryos. We found that PDE5 was barely expressed in early embryos from both species (12 days post coitum, dpc, in the mouse and 7 weeks post-conception, wpc, in the human, 2 embryos/species observed), however discrete areas were positive to anti-PDE5 antibodies either in mouse or in human embryos. In the mouse embryo PDE5 was mainly localized in the mesenchymal tissue included between the dorsal aorta and the anterior gut and in sparse cells within the liver bud (Suppl. Fig. 1A). Similarly to the mouse, positive PDE5 staining was found in the mesenchyme localized between the dorsal aorta and the primitive intestine in the human embryo (Suppl. Fig. 1B). At a later human embryonic stage (12 wpc, 2embryos observed) PDE5 expression was still confined to few tissue types. Strong PDE5 positivity was found in the medial layer of the thoracic and abdominal aorta (included collaterals), pulmonary arteries and gastrointestinal tract, and in sparse polynucleated cells of the liver (Fig. 5A). These cells proved to be megakaryoblasts, as identified by anti-Von Willebrand antibody in in vitro cultures of mouse bone marrow cells (Suppl. Fig. 1D), in agreement with the notion that human platelet are one of the cellular sites of the highest PDE5 activity ${ }^{42}$. Analysis of NOTCH3 revealed that its expression was overlapping that of PDE5 in the inner smooth muscular layer of the dorsal aorta (Fig. 5B) and primitive intestine (Suppl. Fig. 1D) and interestingly, also in megakaryoblasts of the liver (Fig. 5C).

\section{Discussion}

Vascular smooth muscle cells, that physiologically control the vascular tone of the arterial bed, counteract hypertensive stimuli to undergo relaxation by increasing intracellular cyclic GMP and AMP nucleotides. Endogenous vasodilators such as nitric oxide (NO), atrial, brain or type $C$ natriuretic peptides (ANP, BNP and CNP, respectively) control smooth muscle tone through activation of guanylyl cyclase, elevation of cGMP, and activation 

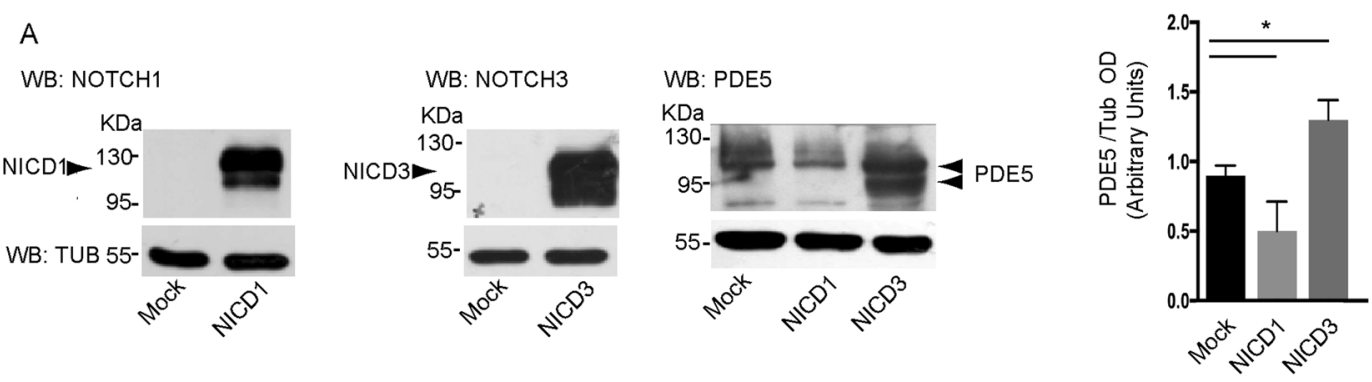

B

U87MG

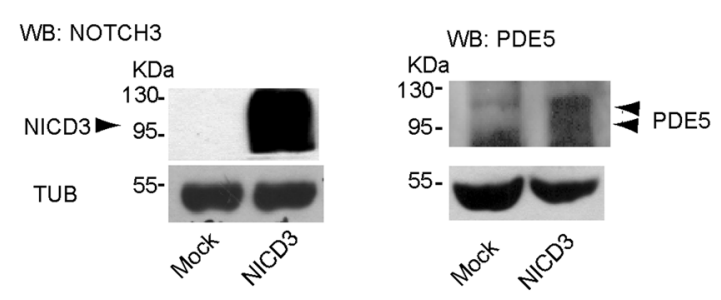

C
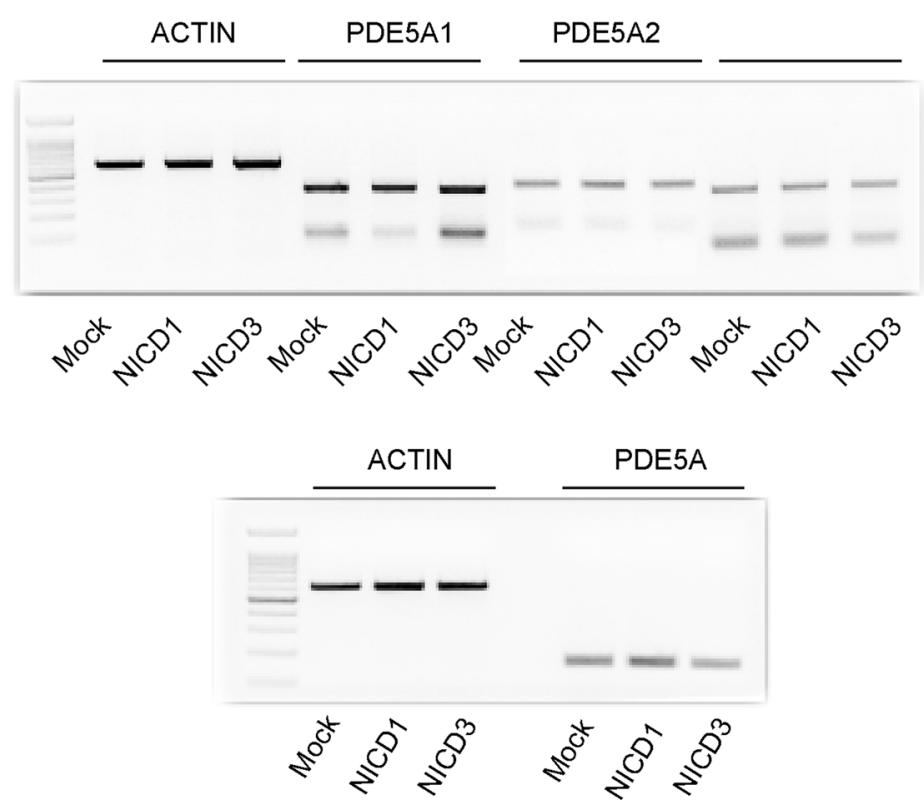

Figure 3. NOTCH3 regulates PDE5 expression. Representative western blot showing decrease of PDE5 protein levels in human VSMCs transfected with NICD1 and increase of PDE5 expression upon NICD3 intracellular domain transfection in human VSMCs (A) and in U87MG cells (B). Bottom panel in (A) represents densitometric analysis on four independent experiments. Bars represent $\pm S D * p<0.05$. Representative semiquantitative RT-PCR showing mRNA levels of total PDE5A, PDE5A1, A2 and A3 isoforms in NICD1 or NICD3 overexpressing human VSMCs $(\mathbf{C})$. Three independent experiments were performed.

of cGMP-dependent protein kinase (PKG). PKG then phosphorylates physiological substrates to reduce intracellular $\mathrm{Ca}^{2+}$ concentration and to desensitize cells to $\mathrm{Ca}^{2+}$, leading to smooth muscle relaxation ${ }^{29}$. PDE5 is the main cGMP-hydrolizing PDE (cGMP-PDE) that lowers cGMP concentration in aortic smooth muscle cells and together with PDE1A, a Calcium-Calmodulin depentent cGMP-PDE, revert the relaxation state ${ }^{29}$. Genetic alterations of factors that regulate the NO/cGMP pathway have been shown to occur in a subset of familiar thoracic aortic aneurysms, indicating that unbalanced cGMP-dependent cascade plays an important role in the generation of aortic wall defects. In support to this evidence is the observation that NO synthase2 (NOS2), one of the five NO synthetic enzymes, is a key mediator of the aortic pathology found in Adamts1 haploinsufficiency and in Marfan syndrome ${ }^{26}$. In these contexts PDE5 may prevent excessive smooth muscle relaxation and aortic dilation resulting from prolonged stimuli that increase cGMP levels and cGMP-activated signaling pathway. 

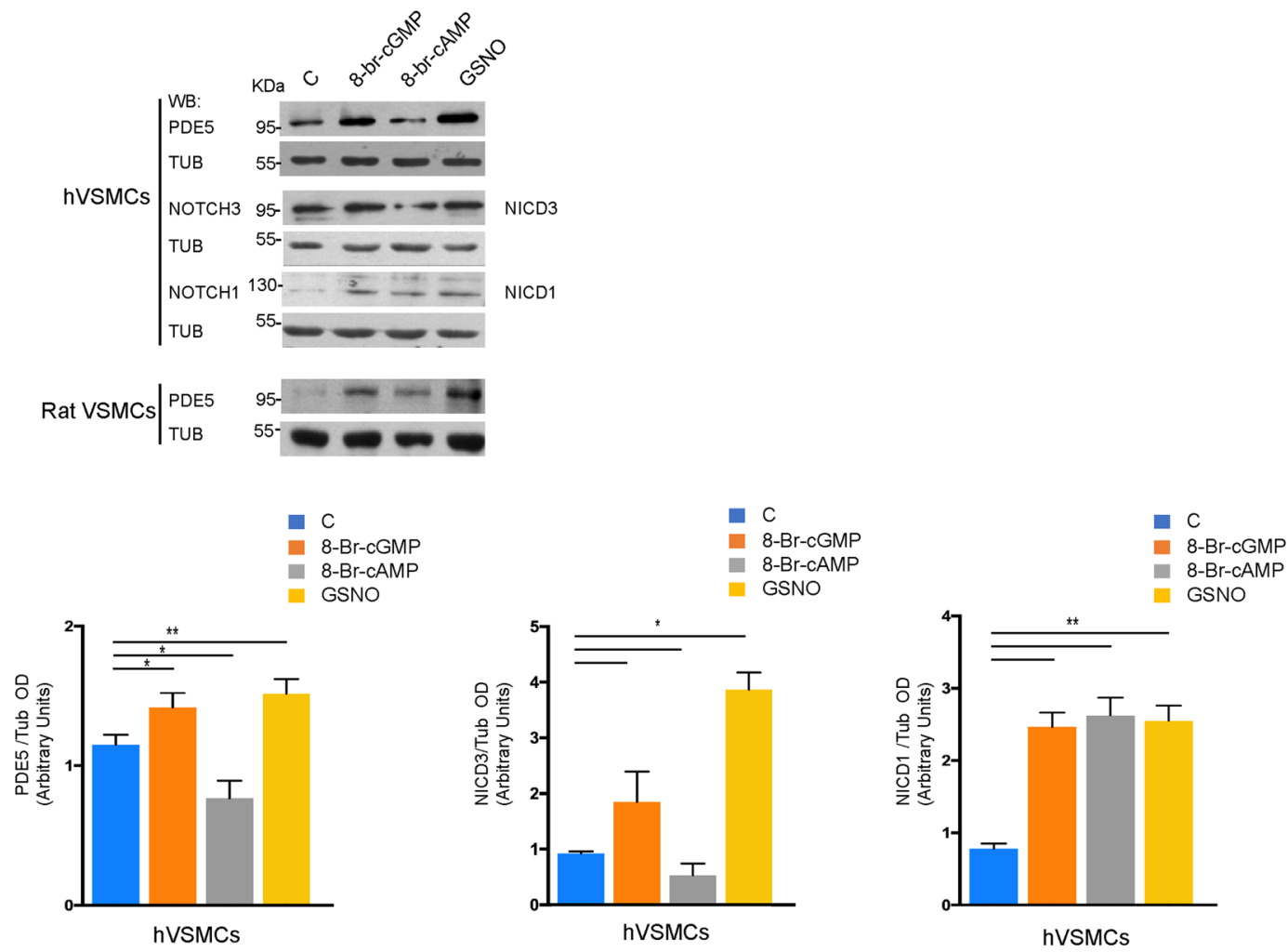

Figure 4. PDE5 expression after cGMP or cAMP stimulation of human and rat VSMCs. Representative western blot showing PDE5, NOTCH1 and NOTCH3 protein levels in human VSMCs (A) and PDE5 protein levels in rat VSMCs $24 \mathrm{hr}$ post treatment with $0.5 \mathrm{mM}$ 8-Br-cGMP, $0.5 \mathrm{mM}$ 8-Br-cAMP and $100 \mathrm{nM}$ GSNO respectively (B). Histograms represent densitometric quantification of western blot analysis in (A). Bars represent \pm SD. $* \mathrm{p}<0.05$ on three experiments.

We previously showed that PDE5 expression in vitro is strongly increased and associated to quiescent, contractile SMCs and its levels are down regulated when VSMCs resume proliferation ${ }^{43}$, suggesting that PDE5 is a marker of the contractile phenotype. In agreement with these evidences, reports have demonstrated that switching of SMCs from the physiological contractile phenotype to a synthetic state associates to aortic aneurysm formation and rupture ${ }^{44}$, suggesting that PDE5 lowering in contractile SMCs might play an important role in the genesis of aortic aneurysms. In line with this hypothesis, here we report that PDE5 protein and mRNA levels are significantly decreased in the wall of Marfan, BAV and TAV aortic aneurysms compared to control aortas, thus associating PDE5 to aortic aneurysm development. The decrease of PDE5 total mRNA levels in aortic aneurysm tissue corresponded to the mRNAs decrease of all the three isoform that, on the contrary, were normally expressed in control aortas.

Our results suggest that PDE5 might play an important role in the regulation of cGMP levels in VSMCs. In mouse cardiomyocytes it has been recently shown that the three PDE5A isoforms had differential subcellular localization, PDE5A1 being mainly localized in the cytoplasm, while mPDE5A2 and mPDE5A3 showing also nuclear distribution ${ }^{30}$. We previously demonstrated that PDE5 is mainly localized in the cytoplasmic compartment and also concentrated within the centrosomes of myometrial SMCs ${ }^{43}$, strongly supporting its role in contraction, and possibly in the control of cell division. The lack of isoform specific antibodies, however, makes it difficult to identify the correct localization of these isoforms in human cells. We found that PDE5 is expressed in the aortic smooth muscle layer of early mouse and human embryos, suggesting that cGMP metabolism during aortic development is active and might play a role in the control of the arterial wall development and function.

Although it has been hypothesized that PDE5 transcripts arise from two different promoters and/or by the splicing of three alternative first exons in the pre-mRNA ${ }^{33}$, very few is known on the regulation of their expression in human tissues. Among the potential factors that could regulate the synthesis of PDE5 mRNA isoforms in smooth muscle cells, we found that components of the NOTCH family of transmembrane/co-transcription factors involved in VSMCs development were differentially expressed in aortic aneurysm samples and that they could influence PDE5 expression in smooth muscle cells. We found that NOTCH3 expression was increased in Marfan syndrome and TAV samples while NOTCH1 was up-regulated only in Marfan syndrome aortas, suggesting that the defects of extracellular matrix structure present in this syndrome can affect NOTCH expression. By overexpressing constitutive active intracellular domains of NOTCH1 or NOTCH3 we observed that they differently modulated PDE5 levels in VSMCs in vitro. While NOTCH1 overexpression induced a decrease of PDE5 levels, NOTCH3 up-regulated PDE5A1 and PDE5A3. The modulation of PDE5 protein expression was not mediated by modification of mRNA levels for none of the isoforms, as indicated by semiquantitative RT-PCR 

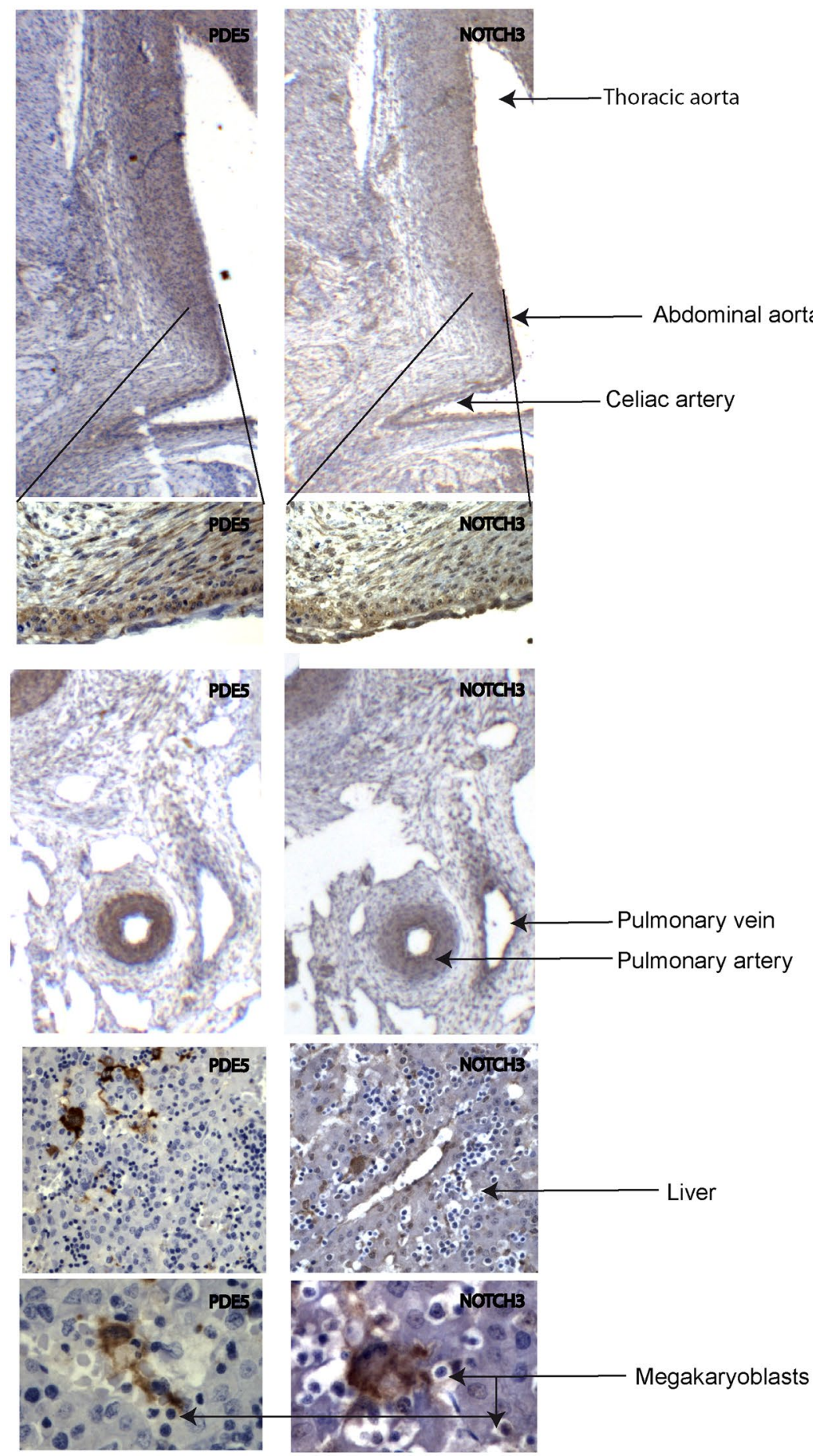

Figure 5. PDE5 and NOTCH3 expression during development of mouse and human fetal aortas.

Representative immunohistochemical evaluation of PDE5 and NOTCH3 on early mouse (12 days post coitum, $\mathrm{dpc}$ ) and human (7 weeks post-conception, wpc) embryos showing positivity for both protein in the medial layer of the dorsal aorta, pulmonary arteries, gastrointestinal tract, and in megakaryoblasts of the liver in both mouse and human embryos. Black arrows indicate thoracic aorta, abdominal aorta, celiac artery, pulmonary vein and pulmonary artery respectively.

experiments. While we do not think that NOTCH1 can control PDE5 expression cell-autonomously in vivo, since it is primarily expressed in the endothelial compartment, our results suggest that NOTCH3 might affect PDE5 expression by controlling mRNA translation of the specific PDE5 isoforms or by affecting protein stability and its degradation. In support to this latter hypothesis, a recent report has identified a PDE5 ubiquination system, based on RhoBTB1, a Cullin3-binding protein that controls PDE5 ubiquitination, its stability and its enzymatic activity ${ }^{45}$. The evidence that NOTCH3 mRNA levels are not modified in BAV compared to control tissues while 
in Marfan syndrome and TAV aortic specimens they are even increased, does not rule out the possibility that NOTCH3 activation by proteolytic cleavage that leads to NOTCH-activated signaling pathway is impaired and/ or not working in the different aneurysmatic pathologies. Moreover, the evidence that PDE5 expression parallels that of NOTCH3 mapping to the same smooth muscle structures, as well as in megakaryocytes, during human embryonic development suggests a link between these two proteins.

Specific PDE5 inhibitors are currently under clinical trial during prenatal life for the treatment of fetal growth restriction (FGR), neonatal pulmonary hypertension or preeclampsia ${ }^{46-48}$ to target vascular smooth muscle layers, thus the safety of these molecules on the development of the aortic wall needs to be warranted. We found that in vitro stimulation of VSMCs with a membrane-permeable cGMP analog up-regulates PDE5 levels, indicating that a negative feedback control tightly monitors cGMP levels in smooth muscle cells. On the contrary, increased intracellular cAMP concentrations decrease PDE5 levels, potentially amplifying the mio-relaxant effects of cAMP. The same effects of cGMP and cAMP were observed to regulate activated NICD3 levels, suggesting that malfunction of cGMP/NICD3/PDE5 loop and excessive VSMC relaxation could contribute to aortic aneurysm formation. In conclusion, we suggest that PDE5 expression and activity might play a protective role in the NO/cGMP/PKG axis by controlling the vascular tone of SMCs. It will be interesting to verify this hypothesis on Pde5 KO animal models.

\section{Materials and Methods}

Populations. This study protocol was approved by the institutional review board at University of Rome TorVergata. It included 38 TAA individuals (7 patients with Marfan's Syndrome, 19 TAV patients and 12 BAV patients). They were admitted in the Cardiac Surgery Unit of Tor Vergata University from July 2017 to December 2018 for ascending aorta surgical procedure. Control aortas without aortic pathologies were obtained from organ donors from the organ transplantation center (University of Torvergata). To match the age of control donors we chose 2 young donors ( 37 and 45 years of age, male and female, respectively) and from 2 older donors (68 and 78 years of age male and female, respectively) to match them with the patient age. Our study received approval from local ethic committees and all participants gave their informed consent. Data were encoded to ensure patient and control protection. All demographic variables, echocardiographic parameter and operative characteristics are summarized in Table 1.

Embryos, Aortic specimens and histopathological assays. Three human fetuses were obtained from pregnant women who underwent therapeutic abortion at 9-12 weeks of gestation at the Dipartimento di Promozione della Salute, Materno-Infantile, Medicina Interna e Specialistica di Eccellenza "G. D’Alessandro" (PROMISE) of the University of Palermo. The ethical committee of the Department of Biomedicine, Neuroscience and Advanced Diagnostics of the University of Palermo, Italy approved the use of human fetal tissue for research purpose. The study was performed in accordance with the relevant guidelines and regulations. Informed, written consent was obtained from all patients. Mouse embryos were obtained from CD-1 pregnant females at 12 and 15 days post coitum $(\mathrm{dpc})$. All experiments were performed in compliance with the Tor Vergata University Institutional Animal Care and all the procedures adhered to the standards published in Guide for the Care and Use of Laboratory Animals. Experimental procedures involving mice were approved by the Italian Ministry of Health. For immunolocalization studies, human and mouse fetal tissues were fixed in formalin and embedded in paraffin. Immunohistochemistry was performed on $5 \mu \mathrm{m}$ sections. For PDE5A detection, de-paraffinized and rehydrated sections were retrieved in TE solution ( $\mathrm{pH}$ 9). Endogenous peroxidase was blocked (ScyTek, Logan, UT) and then sections were incubated for $1 \mathrm{~h}$ at room temperature with rabbit polyclonal antibody anti-PDE5A (1:100 diluition, H-120, sc 32884; Santa Cruz Biotech, Heidelberg, Germany). This antibody has been raised against aminoacids 31-150 in the N-terminus of human PDE5, a region shared by PDE5A1, A2 and A3, and it is potentially able to recognize all the three PDE5 isoforms. NOTCH3 and VWF antigens were retrieved by microwave (10 min in EDTA buffer) and detection was performed with rabbit anti-NOTCH3 (1:100, cod. 5276, Cell Signaling, Milan, Italy) anti-VWF(A0082, Dako-Agilent, Milan, Italy). Retrieval HRP AntiPolyvalent Lab Pack (Scytek Laboratories, Utah, USA), (Alexa Fluor ${ }^{\circledR}$ 594, Thermofisher, Milan, Italy, 1:4000). DAB (Scytek Laboratories, Utah, USA) was used as chromogen in immunohistochemistry experiments. Sections were counterstained with Harris's hematoxylin or DAPI. Morphology of control and TAA samples was performed by hematoxylin-eosin (H\&E). For each control and patient aortic section, at least three immunostaining reactions were performed and four regions of interest (ROI) analysed. Control negative immunostainings were performed by omitting the primary antibody (see Suppl. Fig. 1E). Densitometric analyses were performed by ImageJ software analysis.

For RNA extraction, aortas were cut in squares of about $1 \mathrm{~cm} \times 1 \mathrm{~cm}$ and snap frozen. Tissues or cultured cells were extracted with Trizol 1 reagent (Invitrogen). RNA was DNAase treated (RQ1 DNAase, Promega) and reverse transcribed with random hexamer primers using an Invitrogen cDNA reverse transcription kit (with a reverse transcriptase-negative control) cells by Quantitative real-time PCR was performed using SsoAdvanced ${ }^{\mathrm{TM}}$ Universal SYBR ${ }^{\circledR}$ Green Supermix (Biorad) on an Applied Biosystems 7300 Real Time Machine (Applied Biosystems). Relative expression was calculated using the $2^{-\mathrm{DDCt}}$ method. CT means from samples for each condition were pooled and standard deviation (SD) was calculated.

Western blotting was performed on whole cell extracts obtained following cell lysis in $10 \mathrm{mM}$ HEPES $\mathrm{pH}$ 7.9, $1 \%$ Triton X-100, $10 \mathrm{mM} \mathrm{KCl,} 1.5 \mathrm{mM} \mathrm{MgCl} 2,0.1 \mathrm{mM}$ EGTA, $0.5 \mathrm{mM}$ dithiothreitol (DTT), $10 \mathrm{mM}$ $\beta$-glycerophosphate, $0.1 \mathrm{mM}$ sodium vanadate, and protease inhibitor cocktail (Sigma-Aldrich, Milan, Italy). Rabbit anti-NOTCH1 (ab65297, Abcam, Cambridge, UK), rabbit anti-NOTCH3 and mouse anti-Tubulin (T4026, Sigma-Aldrich) antibodies were diluted 1:1000. Signals were detected with HRP-conjugated secondary antibodies and enhanced chemiluminescence (Santacruz Biotech). Western blots were performed on three independent experiments. 


\begin{tabular}{|c|c|c|c|c|}
\hline Variables & Marfan Syndrome $(n=7)$ & Tricuspid Valve $(\mathrm{n}=19)$ & Bicuspid Valve $(n=12)$ & p-value \\
\hline BSA & $1.9[1.2,2.4]$ & $1.9[1.5,3.3]$ & $1.9[1.5,2.1]$ & 0,875 \\
\hline BMI & $21.9(5)^{*}$ & $28.5(4.3)^{*}$ & $25.8(2.5)$ & 0,002 \\
\hline Age & $35.9(14.5)^{*}$ & $69.4(10.5)^{*}$ & $61(12.9)^{*}$ & $<0.001$ \\
\hline Gender M & $4(57.1 \%)$ & $13(68.4 \%)$ & $10(83.3 \%)$ & 0,357 \\
\hline Hypertension & $5(71.4 \%)$ & $13(68.4 \%)$ & $10(83.3 \%)$ & 0,628 \\
\hline Familiarity for cardiovascular diseases & $7(100 \%)$ & $14(73.6 \%)$ & $9(75 \%)$ & 0,438 \\
\hline \multicolumn{5}{|l|}{ Echocardiographyc parameter } \\
\hline Aortic root & $50[44,55]$ & $47[32,53]$ & $44.5[35,50]$ & 0,078 \\
\hline Ascending aorta & $47.4(6.8)$ & $50.4(3.7)$ & $47.3(4.8)$ & 0,152 \\
\hline Left ventricul telediastolic diameter & $51.4(3.2)$ & $51.1(4.8)$ & $50.8(3.2)$ & 0,938 \\
\hline Left ventricul telesistolic diameter & $36[33,40]$ & $36[24,43]$ & $36[28,38]$ & 0,867 \\
\hline Septum & $11[10,12]$ & $11[9,14]$ & $12[10,16]$ & 0,221 \\
\hline Posterior wall & $11(0.8)$ & $11.1(1.7)$ & $12(1.9)$ & 0,259 \\
\hline Left ventricul telediastolic volume & $96.9(29.7)$ & $110.1(20.3)$ & $100.9(22.2)$ & 0,342 \\
\hline Left ventricul telesystolic volume & $53.1(9.1)$ & $53.8(9.4)$ & $47.7(9.8)$ & 0,206 \\
\hline Ejection Fraction $\%$ & $55[55,70]$ & $55[50,60]$ & $58[50,60]$ & 0,885 \\
\hline Mitral regurgitation (I or II) & $5(71.4 \%)$ & $15(78.9 \%)$ & $6(50 \%)$ & 0,269 \\
\hline Aortic stenosis & $0(0 \%)$ & $2(10.5 \%)$ & $6(50 \%)$ & 0,017 \\
\hline Aortic regurgitation & $3(42.8 \%)$ & $9(47.3 \%)$ & $5(41.6 \%)$ & 1,000 \\
\hline Systolic pulmonary arterial pressure & $29.1(6.2)$ & $31.2(6.8)$ & $31.1(5.6)$ & 0,747 \\
\hline \multicolumn{5}{|l|}{ Operative characteristics } \\
\hline Procedure type & & & & 0,280 \\
\hline Bental-De Bono & $7(100 \%)$ & $14(73.6 \%)$ & $11(91.6 \%)$ & \\
\hline Ascending aorta replacement & $0(0 \%)$ & $5(26.3 \%)$ & $1(8.3 \%)$ & \\
\hline Associated procedures, yes & $2(28.5 \%)$ & $5(26.3 \%)$ & $1(8.3 \%)$ & 0,498 \\
\hline Emergency & $0(0 \%)$ & $1(5.2 \%)$ & $2(16.6 \%)$ & 0,568 \\
\hline Type of aortic prostheses & & & & 0,081 \\
\hline mechanical & $7(100 \%)$ & $10(52.6 \%)$ & $7(58.3 \%)$ & \\
\hline bioprosthesis & $0(0 \%)$ & $9(47.4 \%)$ & $5(41.7 \%)$ & \\
\hline Size of aortic prostheses & $23[19,25]$ & $23[19,27]$ & $23[21,25]$ & 0,878 \\
\hline Size of vascular prostheses & $25.9(1.5)$ & $27.3(1.9)$ & $26.7(1.3)$ & 0,166 \\
\hline Cardioplegia & & & & 0,805 \\
\hline Cristalloid & $7(100 \%)$ & $16(16 \%)$ & $11(91.7 \%)$ & \\
\hline Ematic & 0 & $3(15.7 \%)$ & $1(8.3 \%)$ & \\
\hline Surgery time & $310[210,800]$ & $240[160,540]$ & $232[185,393]$ & 0,093 \\
\hline Ejection Fraction \% & $60[35,70]$ & $57[40,60]$ & $60[42,65]$ & 0,537 \\
\hline
\end{tabular}

Table 1. Demographic characteristics, echocardiographic parameter, operative characteristics of the study population.

Cell culture and transfections. Uterine artery VSMCs were derived from young aged (25-35 years old women) myometrial samples obtained as reported $\mathrm{in}^{43}$ and consisted in explants of small fragments $(0.5 \mathrm{~mm}$ squares) cut around the arteriolar regions. VSMC from uterine artery were used instead of aortic VSMCs because they showed a higher replating efficiency (more than ten passages) and a higher transfection efficiency (our unpublished results and ${ }^{49}$ ) but a lower infection efficiency with respect to tumor cell lines. The glioblastoma cell lines U87MG and T98G and Hek293T cells were cultured in DMEM 10\%FCS. We included U87MG cells, that do not express PDE5, to show that PDE5 upregulation by NICD3 overexpression was not limited to VSMCs. To validate anti-PDE5 antibodies, T98G cells, that express high levels of PDE5, were infected with silencing vectors (sh-GFP as mock and sh-PDE5 as PDE5 silencing) as previously reported in $^{50}$. Briefly, lentiviral PDE5 silencing vectors [RHS4430-101103886 Clone V3LHS_325328 (silencing sequence: CGGTTAATGCAGAAGTTG) sh-PDE5] and lentiviral scrambled control [RHS2349, sh-GFP] (Dharmacon, Milan, Italy) were transfected in Hek293T cells and all the lentiviral particles obtained were used undiluted for T98G infection.

Rat corpora cavernosa (RCC) smooth muscle cells (gift from E. Carosa) were obtained and cultured as reported in ${ }^{51}$. To prove that megakaryoblasts are the cell types in the fetal liver that express PDE5, we isolated them from mouse femoral bone marrow and cultured in Megacult medium (cat. 04961, Stem Cell Technologies, Cambridge, UK). 8-Br-cGMP, 8-Br-cAMP and GSNO were from Sigma-Aldrich (B1381, B7880 and N4148, respectively).

NICD1-pcDNA3 or NICD3-pcDNA3 plasmids (kind gift of prof. I. Screpanti) encoding for the intracellular domain (ICD) of each NOTCH protein or pcDNA3-empty plasmid (Mock), were transfected in uterine artery smooth muscle cells, using lipofectamine 2000 transfection reagent (Invitrogen, Milan, Italy). 
Oligonucleotides. hPDE5 A1 For 5'-CGATCACTGGGACTTTACCT; hPDE5 A2 For 5'-TGC TATGTTGCCCTTT GGAG; hPDE5 A3 For 5'-AACATGACGGAACCTTGCCA; qPCR-hPDE5 A 1-2-3 Rev 5' - TTATCTGCACGAGGACTCTG; hPDE5 A1-2-3 Rev 5'-GAGCACTG GTCCCCTTCAT; hSMA For 5- ATGTACCCAGGCATTGCTG A; hSMA Rev 5' TTGCTGAT CCACATCTGCTG; hGAPDH For 5'AGGTCGGAGTCAAC GGATTT; hGAPDH Rev 5'-GTGATGGCATGGACTGTGGT. hNOTCH3 For 5'-GAATT GTGAAGTGAACGTGGA; hNOTCH3 Rev 5'-TGGCACA GTCATCGATATTCT; hNOTCH1 For 5'-TATTGACGACGTTGCCGGGTA; hNOTCH1 Rev 5' ATAGTCCTCGGATTG CCTG CACT.

Statistical analysis. All analyses were performed with STATA 14.1 software. All categorical variables are reported as frequencies and percentages, while quantitative variables have been synthesized as mean and standard deviation or median, minimum and maximum. The Fisher test for qualitative variables and the Kruskal-Wallis test or the one-way ANOVA technique for quantitative variables was calculated. The hypotheses of normality and homoschedasticity have been verified through the Shapiro-Wilk test and the Bartlett test. All the tests were considered significant for relative values $\mathrm{p}<0.05$. Furthermore, odds ratios (OR) with $95 \%$ confidence intervals (CI) and their significance were calculated.

\section{References}

1. Criado, F. J. Aortic dissection: a 250-year perspective. Tex Heart Inst J 38, 694-700 (2011).

2. Erbel, R. et al. 2014 ESC Guidelines on the diagnosis and treatment of aortic diseases: Document covering acute and chronic aortic diseases of the thoracic and abdominal aorta of the adult. The Task Force for the Diagnosis and Treatment of Aortic Diseases of the European Society of Cardiology (ESC). Eur Heart J 35, 2873-2926, https://doi.org/10.1093/eurheartj/ehu281 (2014).

3. Sampson, U. K. et al. Global and regional burden of aortic dissection and aneurysms: mortality trends in 21 world regions, 1990 to 2010. Glob Heart 9, 171-180 e110, https://doi.org/10.1016/j.gheart.2013.12.010 (2014).

4. El-Hamamsy, I. \& Yacoub, M. H. Cellular and molecular mechanisms of thoracic aortic aneurysms. Nat Rev Cardiol 6, 771-786, https://doi.org/10.1038/nrcardio.2009.191 (2009).

5. Elefteriades, J. A. Thoracic aortic aneurysm: reading the enemy's playbook. Curr Probl Cardiol 33, 203-277, https://doi.org/10.1016/j. cpcardiol.2008.01.004 (2008).

6. Tromp, G., Kuivaniemi, H., Hinterseher, I. \& Carey, D. J. Novel genetic mechanisms for aortic aneurysms. Curr Atheroscler Rep 12, 259-266, https://doi.org/10.1007/s11883-010-0111-x (2010).

7. Balistreri, C. R. Genetic contribution in sporadic thoracic aortic aneurysm? Emerging evidence of genetic variants related to TLR4-mediated signaling pathway as risk determinants. Vascul Pharmacol 74, 1-10, https://doi.org/10.1016/j.vph.2015.09.006 (2015).

8. Albornoz, G. et al. Familial thoracic aortic aneurysms and dissections-incidence, modes of inheritance, and phenotypic patterns. Ann Thorac Surg 82, 1400-1405, https://doi.org/10.1016/j.athoracsur.2006.04.098 (2006).

9. Guo, D. C. et al. Familial thoracic aortic aneurysms and dissections: identification of a novel locus for stable aneurysms with a low risk for progression to aortic dissection. Circ Cardiovasc Genet 4, 36-42, https://doi.org/10.1161/CIRCGENETICS.110.958066 (2011).

10. Guo, D. C. et al. Mutations in smooth muscle alpha-actin (ACTA2) lead to thoracic aortic aneurysms and dissections. Nat Genet 39, 1488-1493, https://doi.org/10.1038/ng.2007.6 (2007).

11. Zhu, L. et al. Mutations in myosin heavy chain 11 cause a syndrome associating thoracic aortic aneurysm/aortic dissection and patent ductus arteriosus. Nat Genet 38, 343-349, https://doi.org/10.1038/ng1721 (2006).

12. Guo, D. C. et al. LOX Mutations Predispose to Thoracic Aortic Aneurysms and Dissections. Circ Res 118, 928-934, https://doi. org/10.1161/CIRCRESAHA.115.307130 (2016).

13. Wang, L. et al. Mutations in myosin light chain kinase cause familial aortic dissections. Am J Hum Genet 87, 701-707, https://doi. org/10.1016/j.ajhg.2010.10.006 (2010).

14. Guo, D. C. et al. Recurrent gain-of-function mutation in PRKG1 causes thoracic aortic aneurysms and acute aortic dissections. Am J Hum Genet 93, 398-404, https://doi.org/10.1016/j.ajhg.2013.06.019 (2013).

15. Barbier, M. et al. MFAP5 loss-of-function mutations underscore the involvement of matrix alteration in the pathogenesis of familial thoracic aortic aneurysms and dissections. Am J Hum Genet 95, 736-743, https://doi.org/10.1016/j.ajhg.2014.10.018 (2014).

16. Bertoli-Avella, A. M. et al. Mutations in a TGF-beta ligand, TGFB3, cause syndromic aortic aneurysms and dissections. J Am Coll Cardiol 65, 1324-1336, https://doi.org/10.1016/j.jacc.2015.01.040 (2015).

17. Boileau, C. et al. TGFB2 mutations cause familial thoracic aortic aneurysms and dissections associated with mild systemic features of Marfan syndrome. Nat Genet 44, 916-921, https://doi.org/10.1038/ng.2348 (2012).

18. Loeys, B. L. et al. Aneurysm syndromes caused by mutations in the TGF-beta receptor. $N$ Engl J Med 355, 788-798, https://doi. org/10.1056/NEJMoa055695 (2006).

19. Mizuguchi, T. et al. Heterozygous TGFBR2 mutations in Marfan syndrome. Nat Genet 36, 855-860, https://doi.org/10.1038/ng1392 (2004).

20. Pannu, H. et al. Mutations in transforming growth factor-beta receptor type II cause familial thoracic aortic aneurysms and dissections. Circulation 112, 513-520, https://doi.org/10.1161/CIRCULATIONAHA.105.537340 (2005).

21. Regalado, E. S. et al. Exome sequencing identifies SMAD3 mutations as a cause of familial thoracic aortic aneurysm and dissection with intracranial and other arterial aneurysms. Circ Res 109, 680-686, https://doi.org/10.1161/CIRCRESAHA.111.248161 (2011).

22. Mohamed, S. A. et al. Novel missense mutations (p.T596M and p.P1797H) in NOTCH1 in patients with bicuspid aortic valve. Biochem Biophys Res Commun 345, 1460-1465, https://doi.org/10.1016/j.bbrc.2006.05.046 (2006).

23. McKellar, S. H. et al. Novel NOTCH1 mutations in patients with bicuspid aortic valve disease and thoracic aortic aneurysms. J Thorac Cardiovasc Surg 134, 290-296, https://doi.org/10.1016/j.jtcvs.2007.02.041 (2007).

24. Kuang, S. Q. et al. FOXE3 mutations predispose to thoracic aortic aneurysms and dissections. J Clin Invest 126, 948-961, https://doi. org/10.1172/JCI83778 (2016).

25. Awad, M. A., Aldosari, S. R. \& Abid, M. R. Genetic Alterations in Oxidant and Anti-Oxidant Enzymes in the Vascular System. Front Cardiovasc Med 5, 107, https://doi.org/10.3389/fcvm.2018.00107 (2018).

26. Oller, J. et al. Nitric oxide mediates aortic disease in mice deficient in the metalloprotease Adamts1 and in a mouse model of Marfan syndrome. Nat Med 23, 200-212, https://doi.org/10.1038/nm.4266 (2017).

27. Kelly, P. Targeting nitric oxide to treat aneurysm. Science 355, 491-492, https://doi.org/10.1126/science.355.6324.491-c (2017).

28. Zhang, W. et al. Exome sequencing reveals a de novo PRKG1 mutation in a sporadic patient with aortic dissection. BMC Med Genet 19, 218, https://doi.org/10.1186/s12881-018-0735-1 (2018).

29. Rybalkin, S. D., Yan, C., Bornfeldt, K. E. \& Beavo, J. A. Cyclic GMP phosphodiesterases and regulation of smooth muscle function. Circ Res 93, 280-291, https://doi.org/10.1161/01.RES.0000087541.15600.2B (2003).

30. Campolo, F. et al. Identification of murine phosphodiesterase $5 \mathrm{~A}$ isoforms and their functional characterization in HL-1 cardiac cell line. J Cell Physiol 233, 325-337, https://doi.org/10.1002/jcp.25880 (2018). 
31. Lin, C. S., Lau, A., Tu, R. \& Lue, T. F. Identification of three alternative first exons and an intronic promoter of human PDE5A gene. Biochem Biophys Res Commun 268, 596-602, https://doi.org/10.1006/bbrc.2000.2186 (2000).

32. Kotera, J., Fujishige, K. \& Omori, K. Immunohistochemical localization of cGMP-binding cGMP-specific phosphodiesterase (PDE5) in rat tissues. J Histochem Cytochem 48, 685-693, https://doi.org/10.1177/002215540004800512 (2000).

33. Lin, C. S., Lau, A., Tu, R. \& Lue, T. F. Expression of three isoforms of cGMP-binding cGMP-specific phosphodiesterase (PDE5) in human penile cavernosum. Biochem Biophys Res Commun 268, 628-635, https://doi.org/10.1006/bbrc.2000.2187 (2000).

34. Gridley, T. Notch signaling in vascular development and physiology. Development 134, 2709-2718, https://doi.org/10.1242/ dev.004184 (2007).

35. Balistreri, C. R. et al. Deregulation of Notch1 pathway and circulating endothelial progenitor cell (EPC) number in patients with bicuspid aortic valve with and without ascending aorta aneurysm. Sci Rep 8, 13834, https://doi.org/10.1038/s41598-018-32170-2 (2018).

36. Proost, D. et al. Performant Mutation Identification Using Targeted Next-Generation Sequencing of 14 Thoracic Aortic Aneurysm Genes. Hum Mutat 36, 808-814, https://doi.org/10.1002/humu.22802 (2015).

37. Charles, N. et al. Perivascular nitric oxide activates notch signaling and promotes stem-like character in PDGF-induced glioma cells. Cell Stem Cell 6, 141-152, https://doi.org/10.1016/j.stem.2010.01.001 (2010).

38. Domenga, V. et al. Notch3 is required for arterial identity and maturation of vascular smooth muscle cells. Genes Dev 18, 2730-2735, https://doi.org/10.1101/gad.308904 (2004).

39. Krebs, L. T. et al. Notch signaling is essential for vascular morphogenesis in mice. Genes Dev 14, 1343-1352 (2000).

40. Joutel, A. et al. Notch3 mutations in CADASIL, a hereditary adult-onset condition causing stroke and dementia. Nature 383, 707-710, https://doi.org/10.1038/383707a0 (1996).

41. Goudie, A. R., Lipworth, B. J., Hopkinson, P. J., Wei, L. \& Struthers, A. D. Tadalafil in patients with chronic obstructive pulmonary disease: a randomised, double-blind, parallel-group, placebo-controlled trial. Lancet Respir Med 2, 293-300, https://doi.org/10.1016/ S2213-2600(14)70013-X (2014).

42. Hidaka, H. \& Asano, T. Human blood platelet 3': $5^{\prime}$-cyclic nucleotide phosphodiesterase. Isolation of low-Km and high-Km phosphodiesterase. Biochim Biophys Acta 429, 485-497 (1976).

43. Dolci, S. et al. Subcellular localization and regulation of type-1C and type-5 phosphodiesterases. Biochem Biophys Res Commun 341, 837-846, https://doi.org/10.1016/j.bbrc.2006.01.035 (2006).

44. Wang, L. et al. Association of smooth muscle cell phenotypes with extracellular matrix disorders in thoracic aortic dissection. J Vasc Surg 56, 1698-1709, 1709 e1691, https://doi.org/10.1016/j.jvs.2012.05.084 (2012).

45. Mukohda, M. et al. RhoBTB1 protects against hypertension and arterial stiffness by restraining phosphodiesterase 5 activity. J Clin Invest 130, 2318-2332, https://doi.org/10.1172/JCI123462 (2019).

46. Sharp, A. et al. Maternal sildenafil for severe fetal growth restriction (STRIDER): a multicentre, randomised, placebo-controlled, double-blind trial. Lancet Child Adolesc Health 2, 93-102, https://doi.org/10.1016/S2352-4642(17)30173-6 (2018).

47. Farrow, K. N. \& Steinhorn, R. H. Phosphodiesterases: emerging therapeutic targets for neonatal pulmonary hypertension. Handb Exp Pharmacol, 251-277, https://doi.org/10.1007/978-3-642-17969-3_11 (2011).

48. Trapani, A. Jr. et al. Perinatal and Hemodynamic Evaluation of Sildenafil Citrate for Preeclampsia Treatment: A Randomized Controlled Trial. Obstet Gynecol 128, 253-259, https://doi.org/10.1097/AOG.0000000000001518 (2016).

49. Zhang, C. et al. Regulation of vascular smooth muscle cell proliferation and migration by human sprouty 2. Arterioscler Thromb Vasc Biol 25, 533-538, https://doi.org/10.1161/01.ATV.0000155461.50450.5a (2005).

50. Cesarini, V. et al. Type 5 phosphodiesterase regulates glioblastoma multiforme aggressiveness and clinical outcome. Oncotarget 8 , 13223-13239, https://doi.org/10.18632/oncotarget.14656 (2017).

51. Carosa, E. et al. Platelet-derived growth factor regulation of type-5 phosphodiesterase in human and rat penile smooth muscle cells. J Sex Med 11, 1675-1684, https://doi.org/10.1111/jsm.12568 (2014)

\section{Acknowledgements}

Authors wish to thank Prof. I. Screpanti (University of Rome La Sapienza, Rome, Italy) for NOTCH1 and NOTCH3 plasmids and dr. E. Carosa (University of L’Aquila, L’Aquila, Italy) for RCC cell line. This work has been supported by MIUR grants 2015XSNA83_006 and 2017ATZ2YK_002 to SD, PRIN 2015XCR88M_001 and FIRB no. RBAP109BLT_004 and to E.A.J.

\section{Author Contributions}

Valeriana Cesarini, Flavia Botti, Giorgio Antonelli, Carmela Balistreri performed PCR experiments, immunohistochemistry and western blot experiments. Gabriele Rossi performed histological analysis. Calogera Pisano and Giovanni Ruvolo performed vascular surgery and handled patient samples. Emmanuele Jannini, Susanna Dolci and Carmela Pisano wrote the manuscript. All the authors discussed the results.

\section{Additional Information}

Supplementary information accompanies this paper at https://doi.org/10.1038/s41598-019-48432-6.

Competing Interests: The authors declare no competing interests.

Publisher's note: Springer Nature remains neutral with regard to jurisdictional claims in published maps and institutional affiliations.

Open Access This article is licensed under a Creative Commons Attribution 4.0 International License, which permits use, sharing, adaptation, distribution and reproduction in any medium or format, as long as you give appropriate credit to the original author(s) and the source, provide a link to the Creative Commons license, and indicate if changes were made. The images or other third party material in this article are included in the article's Creative Commons license, unless indicated otherwise in a credit line to the material. If material is not included in the article's Creative Commons license and your intended use is not permitted by statutory regulation or exceeds the permitted use, you will need to obtain permission directly from the copyright holder. To view a copy of this license, visit http://creativecommons.org/licenses/by/4.0/.

(c) The Author(s) 2019 